\title{
Los vacíos de la planeación participativa en la formulación de los planes de desarrollo local en Bogotá
}

\author{
Os vazios do planejamento participativo na formulação de planos de \\ desenvolvimento local em Bogotá
}

The vacuums of participatory planning in the formulation of local development plans in Bogotá

Naidú Duque-Cante[a] (iD)

[a] Magíster en Administración Pública. Profesora de planta de la Institución Universitaria Politécnico Grancolombiano (IUPG), Faculdad de Negocios, Gestión y Sostenibilidad, Programa de Administración Pública, Bogotá, Distrito Capital, Colombia

Cómo citar: Duque-Cante, N. (2019). Los vacíos de la planeación participativa en la formulación de los planes de desarrollo local en Bogotá. urbe. Revista Brasileira de Gestão Urbana, v.11, e20180193. https://doi.org/10.1590/21753369.011.e20180193

\section{Resumen}

La participación ciudadana constituye actualmente uno de los insumos más importantes para orientar los procesos de planeación del sector público, cuyo propósito es lograr una orientación de los recursos que se ajuste mejor a la solución de los problemas más significativos de la sociedad. Para ello, Colombia cuenta con marcos normativos, para los diferentes niveles territoriales y administrativos, encaminados a regular este proceso, como es el caso particular del Acuerdo 13 de 2000, expedido para guiar la formulación de los planes de desarrollo de las localidades en Bogotá. Sin embargo, a partir del análisis realizado mediante entrevistas semiestructuradas aplicadas a varios actores involucrados en el proceso, se encontró que la puesta en práctica de esta norma, evidencia vacíos y defectos de gran relevancia que ponen en peligro la validez de la participación ciudadana para la planeación local. Estos vacíos se pudieron observar en tres aspectos principales, como son la simultaneidad de la planeación distrital y local, los actores que participan en el proceso y los mecanismos utilizados para recoger las iniciativas ciudadanas, y que se definen a través de metodologías que se elaboran en cada localidad.

Palabras clave: Planeación participativa. Planes de desarrollo. Comunicación participativa. Comunidades. Sociedad civil.

\section{Resumo}

A participação cidadã é atualmente um dos insumos mais importantes para orientar os processos de planejamento do setor público, a fim de obter uma orientação dos recursos que melhor se adapte à solução dos problemas mais significativos da sociedade. Para isso, Colômbia conta com marcos regulatórios, para os diferentes níveis territoriais e administrativos, voltados à regulação desse processo, como é o caso do Acordo 13 de 2000, emitido para orientar a formulação dos planos de desenvolvimento das localidades de Bogotá. No entanto, com base na análise realizada por meio de entrevistas semiestruturadas aplicadas a diversos atores envolvidos no processo, constatou-se que a implementação dessa norma evidencia lacunas e defeitos de grande

NDC és Magíster en Administración Pública de la Escuela Superior de Administración Pública ESAP, e-mail: naidu.duque@gmail.com 
relevância que comprometem a validade da participação cidadã para o planejamento local. Essas lacunas podem ser observadas em três aspectos principais, como a simultaneidade do planejamento distrital e local, os atores envolvidos no processo e os mecanismos usados para coletar as iniciativas dos cidadãos, que são definidos através de metodologias que são desenvolvidas em cada localidade.

Palavras-chave: Planejamento participativo. Planos de desenvolvimento. Comunicação participativa. Comunidades. Sociedade civil.

\section{Abstract}

Citizen participation is currently one of the most important inputs to guide the planning processes of the public sector, in order to achieve an orientation of resources that best fits the solution of the most significant problems of society. For this, Colombia has regulatory frameworks, for the different territorial and administrative levels, aimed at regulating this process, as is the case of Agreement 13 of 2000, issued to guide the formulation of the development plans of the localities in Bogotá. However, based on the analysis carried out through semistructured interviews applied to several actors involved in the process, it was found that the implementation of this norm, evidences gaps and defects of great relevance that endanger the validity of citizen participation for the local planning These gaps could be observed in three main aspects, such as the simultaneity of the district and local planning, the actors involved in the process and the mechanisms used to collect citizens' initiatives, and which are defined through methodologies that are elaborated in each locality.

Keywords: Citizen participation. Planning. Citizenship. Participatory communication. Communities. Civil society.

\section{Introducción ${ }^{1}$}

De acuerdo con el artículo 1ro de la Constitución Política de 1991, Colombia es una república unitaria, descentralizada territorialmente y con autonomía de sus entidades territoriales, las cuales, de acuerdo con el artículo 286 son los departamentos, los cuales se componen, a su vez, de municipios y distritos. En este diseño territorial, los distritos son entidades territoriales de nivel local que por su importancia política, cultural, histórica o económica dejaron de ser municipios para recibir un estatus especial. La ciudad de Bogotá, reconocida como distrito en el año 1945 (acto legislativo 01) es el distrito más antiguo de Colombia y se rige por una norma particular que es el Decreto Ley 1421 de 1993, el cual regula su régimen político administrativo interno, a diferencia de los demás distritos que se rigen mediante la Ley 1617 de 2013.

Debido a la importancia política de Bogotá que la llevaron a ser capital de la república y del departamento de Cundinamarca, la ciudad se ha enfrentado históricamente a condiciones económicas, sociales y territoriales que han exigido un manejo particular de sus asuntos internos. Aunque en Colombia contamos además de la capital con varias ciudades importantes como Medellín, Cali o Barranquilla, Bogotá es la ciudad que más aporta al PIB nacional y la que tiene la mayor concentración de población. De acuerdo con el último Informe de Bogotá Cómo Vamos (2017) la capital duplicó su PIB en los últimos 20 años, al pasar de 72.362 a 147.080 millones de pesos, y redujo la tasa de inflación en $12 \%$. Con estas cifras, la ciudad además duplica el aporte al PIB que realiza el Departamento de Antioquia, cuya capital es Medellín, el cual se encuentra en segundo lugar después de Bogotá. Este informe muestra también que Bogotá concentra 8.080.734 habitantes, cifra que triplica el número de habitantes de Medellín, la segunda ciudad más grande del país.

Con el fin de facilitar el manejo de los asuntos internos la capital se ha dividido en 20 Localidades $^{2}$ cuya configuración territorial ha permanecido similar desde 1954 cuando se anexaron a la ciudad varios de los municipios vecinos que fueron conurbados. Debido a que no ha habido modificaciones en su configuración, actualmente hay localidades que cuentan con niveles poblacionales que superan el millón de habitantes como son los casos de Suba

\footnotetext{
${ }^{1}$ Este artículo se desarrolló en el marco del Proyecto de Investigación Planeación Participativa en Bogotá ejecutado y financia do en su totalidad por la Institución Universitaria Politécnico Grancolombiano IUPG, Bogotá (Colombia). El proyecto pertenece a la línea de investigación de Administración Pública del grupo de investigación Economía, Derechos y Globalización de la IUPG.

2 Localidades de Bogotá: Suba, Kennedy, Engativá, Ciudad Bolívar, Bosa, Usaquén, Fontibón, San Cristóbal, Rafael Uribe Uribe, Usme, Barrios Unidos, Puente Aranda, Tunjuelito, Teusaquillo, Chapinero, Antonio Nariño, Santa Fe, Los Mártires, La Candelaria y Sumapaz.
} 
y Kennedy, y localidades que no superan las cien mil personas como son Sumapaz, Candelaria y Mártires (Bogotá Cómo Vamos, 2017). Dado el gran tamaño del territorio $\left(1775 \mathrm{~km}^{2}\right)$ y la población, la capital, además de contar con un plan de desarrollo diseñado y administrado por el gobierno distrital, ha establecido que las localidades deben formular sus propios planes de desarrollo los cuales se construyen con la participación de las autoridades locales, la instancia de participación y las comunidades, en escenarios de diálogo conocidos como Encuentros Ciudadanos (EC).

Para este proceso la ciudad cuenta con el Acuerdo 13 del año 2000, norma expedida por el Concejo Distrital cuya vigencia ha dado lugar a cinco ejercicios de formulación de planes de desarrollo local (2001, 2004, 2008, 2012 y 2016). Sin embargo, una vez realizado un análisis tanto a esta norma y al marco normativo dentro del cual se ubica, así como a su puesta en marcha durante los años 2012 y 2016, se pudo constatar que la misma padece de vacíos importantes que afectan la validez de la participación ciudadana. Tal como se mostrará más adelante, estos vacíos están relacionados con los momentos de la planeación distrital y local, los actores y la forma como participan, y con los medios dispuestos para la identificación de las iniciativas generadas por los ciudadanos.

\section{Marco teórico y conceptual}

La planeación participativa, entendida como el proceso orientado a la "identificación de problemas y potencialidades del presente y a la formulación de apuestas de futuro" (Velásquez, 1986, p.22), mediante el diálogo entre actores sociales y estatales, es bastante reciente tanto en América Latina como en Colombia. Los primeros desarrollos normativos sobre el tema en Colombia se realizaron en los años 80 del siglo pasado (Gómez et al., 2012). Pero fue con la expedición de la Constitución de 1991 que se logró la creación de un número importante de espacios y mecanismos, de los cuales se esperaba que permitieran la entrada de las comunidades políticas en el escenario de las decisiones y la gestión pública.

Desde el origen, la participación ciudadana, y en particular la participación en los procesos de planeación se ha asociado tanto a nivel nacional como internacional con la descentralización y la modernización del Estado (Velásquez \& González, 2004). Su incorporación en el marco institucional buscaba complementar procesos tendientes a transformar la gestión pública, mediante prácticas que ayudaran a mejorar los procesos de planeación y ejecución, pero que también contribuyeran a relegitimar el rol del Estado (Harnecker \& Bartolomé, 2015). El modelo de manejo centralizado de los asuntos públicos, característico de la segunda mitad del siglo XX, y que potenció procesos de decisión e implementación a partir de la visión centrada en los agentes públicos, condujo a una gestión ineficiente de los problemas sociales lo que derivó a su vez en un debilitamiento de la imagen del Estado frente a los ciudadanos (Gómez et al., 2012).

En todos los casos, la lógica de la planeación participativa, reconocida como derecho humano por la ONU en 1948, es la de garantizar que los diversos actores, estatales y sociales aporten sus puntos de vista en la construcción de un proyecto de impacto colectivo. Dicho proyecto debería orientarse a garantizar que los miembros de la sociedad logren un mejor acceso a los bienes y servicios que les permita la realización de los planes de vida tanto de las generaciones presentes como de las futuras (Velásquez \& González, 2004). En estos términos, la participación ciudadana constituye en la actualidad uno de los instrumentos más importantes para la gobernanza y la gobernabilidad, ya que el proceso de consultar el sentir de las comunidades y los diversos actores involucrados en las decisiones públicas debería derivar en una mejor intervención de los asuntos públicos, y también en el fortalecimiento de la credibilidad en los gobernantes y el Estado en general (Prats Catalá, 2001).

Tal como lo plantea el PNUD "la confianza hacia las instituciones, sumado a demandas no satisfechas por abrir espacios significativos de participación e incidencia pública, puede terminar mermando la legitimidad de reformas importantes y agudizando los cuestionamientos al funcionamiento del sistema democrático" (PNUD, 2014, p. 41). Cada vez se ha hecho más necesario pasar de la fórmula tradicional de decisiones públicas tomadas desde los centro políticos o burocráticos para trasladarlas a los escenarios más cercanos a los actores sociales logrando con ello procesos más inclusivos y participativos (PNUD, 2014; Cunill Grau, 2004).

Estas nuevas tendencias han conducido a una inversión en la forma de tomar decisiones que ha conducido a que las mismas se gesten desde la base social para luego ascender en la escala territorial e institucional, en lo que se conoce como planificación multiescalar (Sandoval et al., 2015). En este marco, "la gobernanza como propuesta de diseño y gestión de políticas públicas en cada uno de los niveles del Estado reclama hoy la articulación entre diversos agentes, el diálogo y los acuerdos como prácticas democráticas" (Williner, 2015, p. 202). El propósito es generar 
dinámicas de articulación y coordinación tendientes a resolver los problemas de la sociedad y aprovechar oportunidades de desarrollo.

De acuerdo con Sandoval et al. (2015) la planificación multiescalar, al entenderse como un instrumento de la gobernanza multiescalar debe, situar las decisiones en el nivel más eficaz y próximo posible a los ciudadanos; garantizar una asociación flexible y cooperación horizontal entre territorios con el fin de que se generen iniciativas propias para problemas que pueden ser comunes; romper con la resolución de problemas de manera sectorializada; resolver los desafíos más allá de las fronteras administrativas formales; y finalmente debe construirse a partir de instancias participativas.

\section{Material y métodos}

El análisis propuesto en este estudio buscaba realizar una revisión tanto del diseño normativo que rige la formulación de los planes de desarrollo de las localidades como su puesta en práctica. Para ello se realizó un análisis del Acuerdo 13 de 2000, el cual reglamenta la realización de los EC, que son el espacio establecido para garantizar la recolección de las iniciativas ciudadanas a ser incluidas en los planes de desarrollo locales. Esta norma, a su vez, se revisó a la luz de la Ley 152 de 1994, la cual regula la formulación de los planes de desarrollo de todas las entidades territoriales y la nación, y del Acuerdo Distrital 12 de 1994 que es la norma específica sobre la formulación del plan de desarrollo del Distrito Capital. Tanto el plan de desarrollo distrital como los planes de desarrollo de las localidades se formulan en el primer semestre del primer año de gobierno distrital, y su vigencia es de cuatro años.

En cuanto a la puesta en práctica del proceso, el estudio tuvo lugar durante la realización de los EC en dos de los cinco procesos de planeación local que se han desarrollado en Bogotá, como fueron los que se celebraron durante el primer semestre de los años 2012 y 2016. Para la recolección de la información se aplicaron ochenta y dos (82) entrevistas semiestructuradas con preguntas abiertas y cerradas, que se dirigieron a los cuatro tipos de actores involucrados en el proceso, en las mismas proporciones para los dos años estudiados. En total se realizaron cuarenta (40) encuestas a ciudadanos, catorce (14) a miembros de las Juntas Administradoras Locales (JAL), ocho (8) a alcaldes locales y veinte (20) a miembros de los Consejos de Planeación Local (CPL). Además de las entrevistas semiestructuradas el análisis tomó en cuenta veinte (20) de las metodologías definidas para los EC durante los dos procesos estudiados.

Las preguntas incluidas en las entrevistas buscaron indagar sobre el nivel de conocimientos que tenían los actores participantes acerca de su rol en el proceso de planeación participativa y de los marcos institucionales dentro de los cuales se encuadra dicho proceso, en especial lo que respecta al Acuerdo 13 de 2000. Asimismo, se recogieron las opiniones sobre la pertinencia de estos instrumentos como garantes de la planeación local de la ciudad, así como las virtudes y defectos que se pudieran derivar de su puesta en práctica. Las preguntas fueron construidas tomando como base el marco normativo definido para la ciudad, en términos de actores, roles y propósitos que el mismo ha establecido para la planeación local.

Para el procesamiento de la información recolectada mediante entrevistas se utilizó el software Atlas Ti, el cual está basado en la Teoría Fundamentada, y facilita la sistematización, procesamiento y análisis de información de tipo cualitativo. Dadas las dimensiones de la información recogida el software permitió su fácil clasificación mediante la definición de códigos axiales y códigos abiertos, que permitieron realizar una mejor comprensión de la misma y que aportaron de manera importante en el análisis que se realiza en este artículo. Algunas de las preguntas cerradas fueron ilustradas mediante gráficos y se sustentaron también en preguntas abiertas en los casos en los que se requirió la verificación de las respuestas.

\section{Análisis y resultados}

La información recolectada fue organizada en tres grandes temas para los cuales se mostrarán los resultados y más adelante las conclusiones. Estos temas incluyen los tiempos de la planeación, los actores que participan en la formulación de los planes de desarrollo local y Las estrategias de identificación y selección de iniciativas ciudadanas. 


\section{Los tiempos de la planeación}

De acuerdo con el marco normativo que rige para la planeación en el Distrito Capital (Acuerdo 13 de 2000), la formulación de los Planes de Desarrollo Local se realiza al mismo tiempo que el Plan de Desarrollo Distrital está en proceso de diseño, lo cual sucede durante el primer semestre del primer año de gobierno del Alcalde Mayor (Ley 152 de 1994). Para ello el Acuerdo 13 de 2000 definió plazos específicos relativos a la conformación del CPL, la convocatoria a los ciudadanos a inscribirse a los EC, y la estructuración de la propuesta inicial del plan de desarrollo local. Asimismo, esta norma establece que las localidades deben comenzar los ejercicios de participación ciudadana utilizando un borrador del Plan de Desarrollo Distrital y una vez aprobado éste, deberán ajustar los avances que han realizado a nivel local con el plan definitivo aprobado por el Concejo Distrital. Dado que en el diseño legal las localidades no disponen de funciones específicas distintas a las del Distrito Capital, los ejercicios de planeación participativa se han orientado a prácticamente todos los sectores de intervención pública definidos para la entidad territorial, lo cual ha facilitado la articulación entre los dos tipos de planes.

Sin embargo, debido a que los dos planes se están formulando al mismo tiempo, no es posible mantener una comunicación constante entre las instancias centrales y locales que permita una sincronización en ambos procesos. Por lo que, una vez formulado el plan distrital, las localidades deben reacomodar sus prioridades y las decisiones tomadas con sus comunidades con el fin de lograr la mayor articulación posible con éste. Esta articulación puede ser de forma, pero también de fondo, lo que significa que ciertos proyectos priorizados a nivel local podrían ya estar considerados desde el Distrito. También puede haber propuestas que tendrían que descartarse por no alinearse con las grandes prioridades definidas para la ciudad, o, debido a la falta de acompañamiento por parte de las instancias centrales y de la misma localidad, las comunidades pueden tomar decisiones sobre temas que no deben intervenirse con recursos públicos y al final tienen que ser eliminados de la propuesta, entre otros.

\section{Los actores que participan en la formulación de los planes de desarrollo Local}

El Acuerdo 13 de 2000 establece como actores involucrados en la planeación local al alcalde local (autoridad ejecutiva local), la Junta Administradora Local (órgano colegiado local), el Consejo de Planeación Local (Instancia de participación) y los ciudadanos. Sin embargo, la organización, funcionamiento y desarrollo de los EC, que son el espacio para la identificación de problemáticas y definición de alternativas de solución, mediante el diálogo con las comunidades, queda en manos, enteramente, del Consejo de Planeación Local (CPL). Esta instancia, se encuentra compuesta por representantes de las diferentes organizaciones que conforman la sociedad civil, en materia social, económica, ambiental, etc. Su papel incluye la definición de la metodología de los EC, así como la división del territorio local, la fijación de las fechas y la escogencia de los lugares para la realización de los encuentros. Finalmente, el CPL participa en la dirección de todo el proceso de participación ciudadana en estos espacios y por lo tanto en la práctica es el que consolida el primer borrador del proyecto de plan de desarrollo local que luego entregará al alcalde local.

De acuerdo con la norma el propósito es que las iniciativas ciudadanas se construyan directamente desde las comunidades mediante la dirección de una instancia netamente civil. Por ello las JAL, los alcaldes locales y los organismos centrales no participan del proceso de programación y desarrollo de la planeación participativa, y aunque estas autoridades pueden asistir a los diferentes espacios concernientes al proceso, no tienen permitido complementar el papel de los CPL. A pesar de esto, la mayoría (70\%) de los miembros de CPL consultados consideró que sería pertinente ampliar la participación de estas autoridades tanto de nivel local como distrital. Esto con el fin de lograr una mayor coherencia entre los propósitos institucionales y los intereses ciudadanos.

Lo anterior se justifica en el hecho que una vez cada alcalde local recibe del CPL las iniciativas ciudadanas recogidas en los EC, le corresponde la consolidación de la propuesta inicial del Plan de Desarrollo Local. Luego el alcalde debe regresar la propuesta al CPL para que este realice sus recomendaciones, las cuales serán consideradas antes de que la propuesta definitiva sea remitida a la JAL para su aprobación y se proceda a la expedición del respectivo acuerdo local del Plan de Desarrollo Local. De acuerdo con la norma, las iniciativas surgidas de los EC que sean incluidas en el plan no podrán ser modificadas, lo cual no significa que todas deban ser incluidas.

Adicionalmente, los alcaldes pueden complementar los planes incluyendo proyectos en aquellas temáticas que no encontraron "doliente" en los EC pero que, desde el punto de vista de estas autoridades deberían ser intervenidos por los gobiernos locales, y que para cada localidad pueden ubicarse en sectores distintos. Sin embargo, aunque los 
alcaldes tienen la libertad de incluir y descartar del plan iniciativas surgidas de encuentros, así como propuestas propias de la administración, los distintos gobiernos distritales han tendido a incentivar a las administraciones locales para que construyan sus planes principalmente con iniciativas ciudadanas, tal como lo manifestaron los alcaldes consultados.

No obstante, al revisar la pertinencia de los aportes y la representatividad de la participación ciudadana, se encontró que entre los ciudadanos consultados en los EC de 2012 y 2016 existe un alto nivel de desconocimiento tanto del marco institucional que rige el proceso como del ejercicio práctico del mismo. Como se puede observar en el gráfico No 1 aunque el $49 \%$ de los ciudadanos consultados manifestó haber participado antes en ejercicios similares, más del $70 \%$ desconocen el acuerdo 13 de 2000 y el propósito mismo de los EC y el $87 \%$ no tenían ningún tipo de formación en participación ciudadana.

Estas cifras son coherentes, además, con las que manifestaron los miembros de los CPL y de las JAL consultados, quienes consideraron, en un porcentaje importante (65\%) que dicho desconocimiento es aprovechado, además, por actores de diverso tipo interesados en que ciertas propuestas formen parte del plan de desarrollo. Entre los intereses más sobresalientes que se mencionaron están los intereses políticos, de las JAL y los alcaldes locales que buscan cumplir sus promesas a partir de la canalización de estas propuestas; los intereses institucionales, debido a que las oficinas desconcentradas de los organismos distritales cuando no pueden resolver los requerimientos de los ciudadanos los dirigen a participar en EC, buscando que se les asignen mayores recursos para sus sectores; y los intereses de los contratistas que quieren asegurar que ciertos proyectos, en los que podrían participar, sean aprobados.

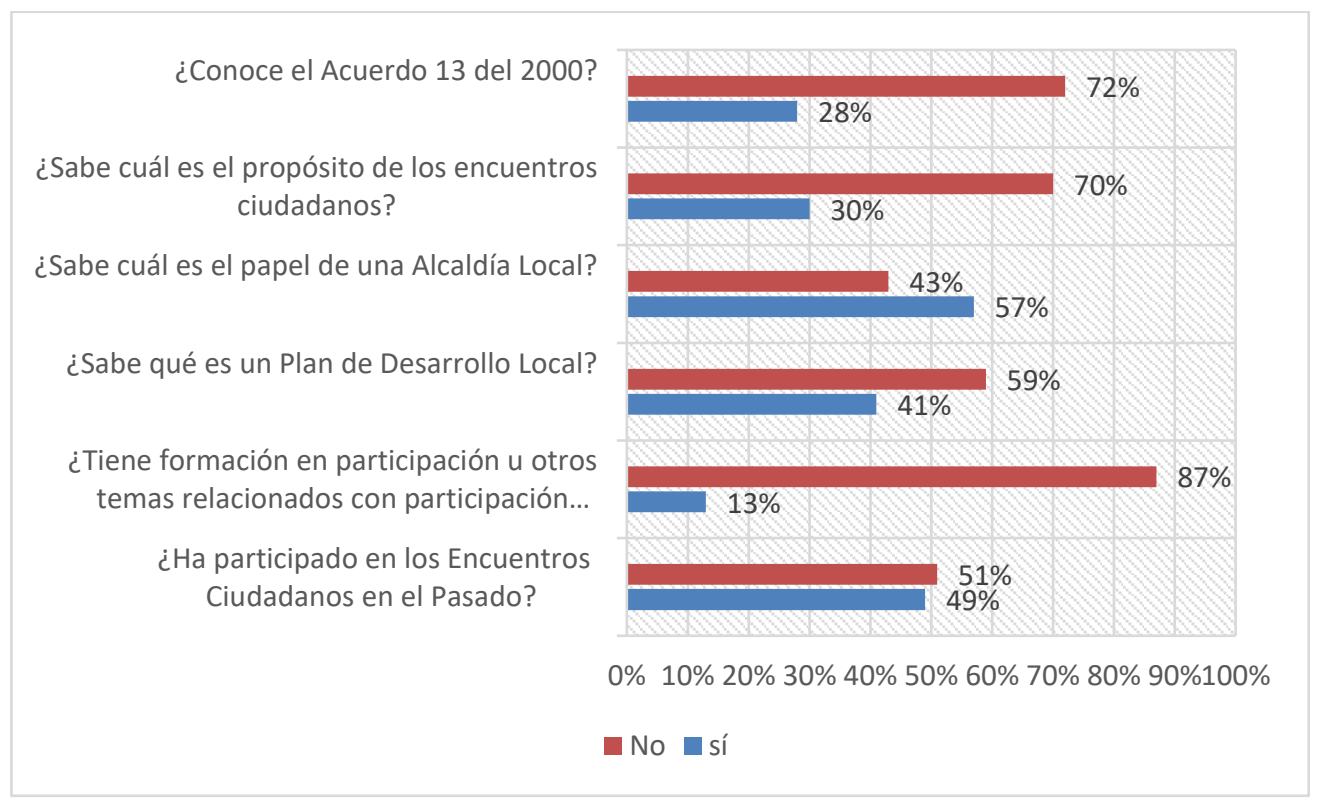

Figura 1 - Nivel de conocimiento de los asistentes a los Encuentros Ciudadanos sobre temas relativos a la planeación participativa en su localidad. Fuente: Autor (2018).

\section{Las estrategias de identificación y selección de iniciativas ciudadanas}

Como se mencionó antes, los CPL tienen la libertad para definir las metodologías que guían los ejercicios de planeación participativa en los EC. Esto ha dado lugar a que entre las diferentes localidades se hayan definido documentos distintos y de gran variedad en cuanto a extensión y contenidos para guiar los ejercicios de planeación participativa. De las 20 metodologías analizadas 10 correspondientes al 2012 y 10 al 2016 se encontraron coincidencias en un $100 \%$ de los casos en cuanto a la incorporación de contenidos orientados a la descripción de los propósitos de los EC y la definición del calendario de los mismos. Sin embargo, aunque en todos los casos los documentos hacen mención de descripción metodológica solo en el $80 \%$ de estos se encontraron indicaciones procedimentales en lo que respecta a la forma como los ciudadanos podían realizar sus aportes en términos de problemas y solamente el $20 \%$ de estos casos incluyeron además metodologías para la priorización de estos problemas. 


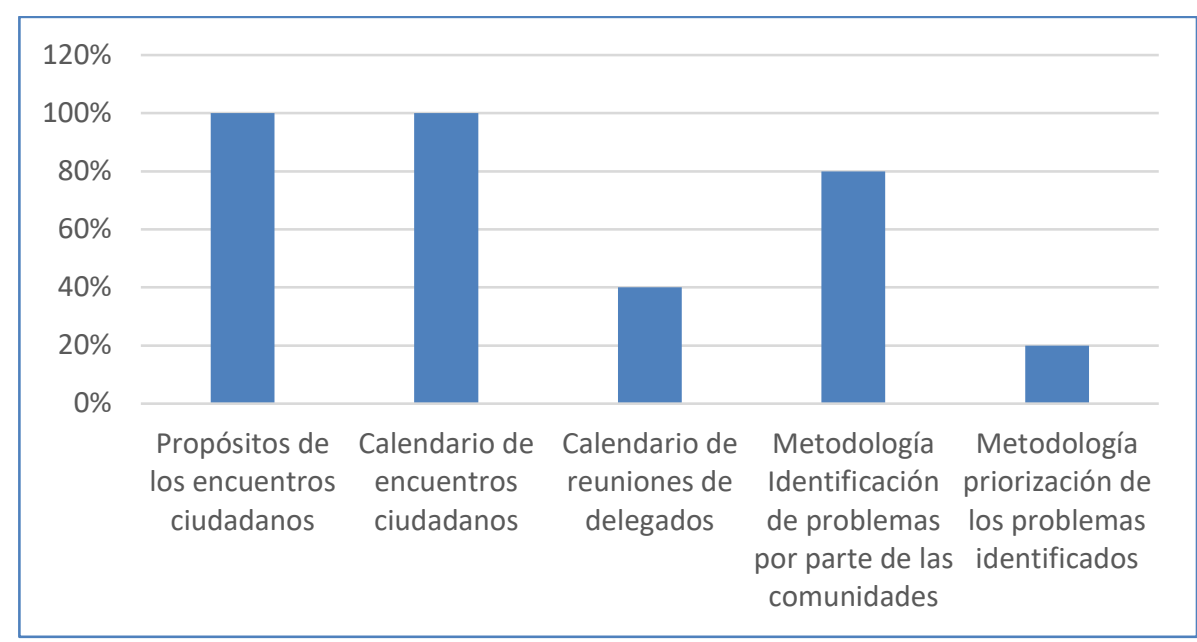

Figura 2 - Contenidos de las metodologías de Encuentros Ciudadanos en 2012 y 2016. Fuente: Autor (2018).

En lo que respecta a las metodologías del año 2012, las descripciones coindicen en todos los casos, con mayor o menor detalle, en prever la organización del proceso en mesas temáticas, en salud, educación, ambiente, vías, etc.; la organización de la participación mediante un moderador y un relator; la participación de los asistentes, mediante el diligenciamiento de una ficha orientadora con o sin formato, donde describir los problemas de la localidad; y, finalmente, se menciona la posibilidad de que los ciudadanos intervengan verbalmente. Asimismo, se prevé la necesidad de seleccionar los problemas más importantes, aunque en la mayoría de los casos no se establece el mecanismo para ello.

Para el caso de las metodologías del año 2016, la diferencia más relevante con respecto al año 2012 es que se presentan mayores desarrollos en términos de la definición de las estrategias a utilizar para organizar la participación, por lo que en la mayoría de los casos se describen metodologías como METAPLAN (7 de las metodologías), seguido de árbol de problemas (2), cartografía social (2) y rotación de las propuestas entre mesas (3). La mayoría de las veces solo se hacen menciones muy generales y solo en un par de casos se describe en detalle el proceso. Además, en varios casos (4) se menciona la combinación de varias metodologías a la vez.

Asimismo, aunque el Acuerdo 13 no lo estipula, en los ejercicios de participación realizados se encontró que del primer o primeros EC se escogieron unos representantes para cada una de las mesas de discusión definidas, quienes hicieron las veces de delegados o comisionados. La función de estos representantes podía variar entre revisar los problemas definidos inicialmente, hacer la revisión de los mismos a la luz de los diagnósticos oficiales, y definir alternativas de solución para los mismos. En otros casos estos ciudadanos simplemente priorizaron los problemas y los volvieron a presentar en los siguientes EC para proceder a la definición de alternativas de solución. No obstante, solo en el $40 \%$ de las metodologías se definieron cronogramas de fechas para la reunión de estos representantes, pero en ninguna de ellas se encontró especificación alguna sobre la función o actividades a realizar en estas reuniones.

Finamente, si bien desde la norma se estableció que los EC deben dar lugar a la generación de iniciativas ciudadanas orientadas a la solución de problemas, no está especificado cómo se debería llegar a este resultado. Dado lo anterior, las metodologías, en el 80\% de los casos llegaron solamente hasta la organización de la participación en los primeros EC y no en todos ellos hasta la priorización de las problemáticas presentadas. Además, en la consulta realizada a los ciudadanos se encontró, que el $30 \%$ no conocía la metodología y el $40 \%$ consideró que no era clara y que los EC se hicieron de forma desordenada.

Debido a que el alcance de los contenidos de las metodologías definidas por los CPL no cubre todo el proceso, lo que sucede entre la definición de las problemáticas iniciales hasta la definición de propuestas a priorizar y el procedimiento de priorización, están sujetos a actividades no planeadas o no programadas tal como lo manifestaron en general los actores consultados. No obstante, en la práctica, se encontró que el proceso final, correspondiente a la priorización de las iniciativas a ser incluidas en el plan se realizó mediante votación de los ciudadanos en las diferentes localidades. 


\section{Discusión y conclusiones}

A partir del análisis realizado fue posible concluir que, aunque la participación ciudadana es considerada, desde la norma, el insumo más importante para planeación del desarrollo en las localidades de Bogotá, la puesta en práctica del proceso presenta defectos importantes que afectan la calidad de esta participación y les restan legitimidad a los aportes de los ciudadanos. Los problemas más importantes están vinculados a la simultaneidad existente entre la planeación distrital y la planeación local; a los actores que participan en el proceso y las condiciones de dicha participación; y a la forma como se realizan los ejercicios participativos que deben derivar en la identificación de las iniciativas ciudadanas a ser incluidas en el proyecto de plan de desarrollo local.

Aunque actualmente se discute la importancia de que en términos de planeación multiescalar lo que pueda realizarse de manera eficiente y efectiva al nivel más cercano a la ciudadanía, se debe abordar en ese nivel (Sandoval et al., 2015), en Bogotá se encontró que la planeación se sigue realizando de arriba hacia abajo. Esto se debe a que los planes locales deben articularse al plan distrital, puesto que las localidades son unidades desconcentradas del Distrito Capital. Esta articulación es posible ya que las localidades no tienen funciones exclusivas distintas a las del distrito (Duque Cante, 2015), por lo que asumen sus responsabilidades dentro de procesos de "delegación, concurrencia, subsidiariedad y complementariedad, de las distintas dependencias de la Administración Central y descentralizada" (Artículo $1^{\circ}$ del Acuerdo 6 de 1992). Como consecuencia las localidades pueden orientar sus procesos de planeación hacia todos los sectores de intervención pública que tengan incidencia en sus territorios, tomando en cuenta las prioridades y las líneas generales de inversión definidas desde el Distrito.

La planeación local, que está referida a propósitos específicos y particulares para cada localidad, debe encajar dentro de la planeación distrital, cuyo propósito es atender los intereses generales para la ciudad, en los mismos sectores. No obstante, en la actualidad los dos tipos de planes se formulan al tiempo, lo cual afecta principalmente a la planeación local, en tanto muchos de los resultados derivados de los ejercicios de planeación participativa se pierden, debiendo ser descartados, principalmente, cuando no se acoplan con los propósitos definidos desde la administración distrital. Dado que no existe una justificación práctica para que los dos procesos de planeación se realicen al tiempo, el correcto engranaje de la planeación local con la distrital debería partir de considerar la existencia de una continuidad en la que primero se formule el plan distrital y posteriormente se realice la planeación local, o viceversa, con lo cual se logre completar la fórmula de la planeación de forma más consistente y ordenada.

En lo que respecta a los actores que participan en el proceso de planeación participativa, el Acuerdo 13 de 2000 considera como actores a las JAL, los CPL, los alcaldes locales y los ciudadanos, pero en la práctica le entrega todo el poder de programación, planeación y dirección de los EC a los CPL, excluyendo de su actuación a instancias y autoridades tanto distritales como locales. Sin embargo, dado el alto grado de libertad que tienen los CPL para llevar a cabo su papel y la falta de claridad que tienen sobre su labor, se evidenció que las metodologías para los EC, que son el principal instrumento que utilizan para guiar los ejercicios de planeación local, adolecen de serios problemas en términos metodológicos y de la estructuración misma del proceso.

En contraste con las propuestas sobre planificación multiescalar en la que se supone que todo proceso participativo debería partir de la existencia, entre otros aspectos de personal capacitado que garantice la rigurosidad del ejercicio (Sandoval et al., 2015), la falta de idoneidad de los miembros del CPL, ha derivado en ejercicios participativos desordenados y con un alto componente de improvisación.

Este tema cobra mayor relevancia cuando se mira desde el punto de vista de los ciudadanos, quienes además de recibir una orientación poco clara e incompleta, tampoco cuentan con la preparación adecuada para enfrentarse a estos escenarios. Los ciudadanos que asisten a los EC, en su mayoría, desconocen la verdadera importancia que tienen los ejercicios de participación ciudadana dentro de la gestión de la localidad y la ciudad. Lo anterior, debido a que no tienen conocimiento del marco normativo más importante que rige la organización y funcionamiento de la ciudad en general, y el que regula los ejercicios de la planeación a nivel local, y tampoco tienen la preparación suficiente para aportar como actores de transformación social.

En términos generales, estamos ante un proceso poco planeado en el que participa una ciudadanía desinformada y con pocas herramientas que le permitan aportar efectivamente en la planeación local. Una planificación efectiva requiere que los ciudadanos que participan en este tipo de ejercicios estén informados de los procesos, los mecanismos y los órganos de participación ciudadana, así como del resultado de las diversas actividades que se desarrollan durante el proceso (Diputació de Barcelona, 2015). En palabras de Velásquez \& González (2003) "la participación puede enriquecer la democracia si se articula a través de mecanismos que combinan información, deliberación y capacidad de intervención de los ciudadanos en los procesos de decisión". 
Esto implica que, además de intervenir la forma y los instrumentos que se utilizan para llevar a cabo los procesos de la planeación participativa, el marco institucional debe considerar la urgente necesidad de realizar ejercicios masivos y permanentes de pedagogía ciudadana. Estos ejercicios, deben tener un alcance generalizado y permear escenarios escolarizados y no escolarizados (Londoño, 2009). Lo anterior con el fin de garantizar una mayor incorporación de los individuos en los asuntos públicos y de contribuir en la comprensión de lo que representa su participación en el funcionamiento del Estado y la administración pública para la gestión del interés colectivo.

De acuerdo con Gómez et al. (2012) un proceso de planeación participativa implica la combinación de elementos de tipo técnico con elementos políticos y pedagógicos "que permitan la comprensión del contexto social y la necesaria viabilidad de lo proyectado" (P.16). El componente político es fundamental, debido a que tanto la materialización de la planeación participativa como la de las decisiones que se derivan de ella están fuertemente ligadas a la voluntad política de los gobernantes de turno (Gómez et al., 2012). Asimismo, la pedagogía ciudadana como estrategia educativa debería garantizar que los participantes en ejercicios de planeación participativa sean conscientes de su rol como transformadores de la realidad social en la que conviven, en tanto miembros de una comunidad (Freire, 1979).

No obstante, el punto de partida debería ser el adecuado diseño normativo y técnico del proceso, que en la actualidad constituye el principal obstáculo de la planeación participativa en las localidades, puesto que no se puede esperar una buena implementación de un proceso mal diseñado. Por este motivo, no fue posible constatar que existieran mejoras en el diseño de los planes locales en la fase de participación ciudadana si comparamos el año 2016 en relación con el año 2012.

Esta situación se evidenció a pesar de que la Alcaldía Distrital ha avanzado en la generación de documentos de orientación para la elaboración del plan distrital y de los planes locales (SDP, 2011), así como de normas administrativas orientadas a fortalecer los procesos de planeación especialmente a nivel local (Alcaldía Mayor, 2016). Aunque estas normas han buscado mejorar la comprensión del proceso por parte de los responsables y dotarlos de herramientas metodológicas, el acuerdo 13 de 2000 que es la norma rectora ha establecido que los CPL son autónomos en la definición de las metodologías y en el diseño y desarrollo de todo el proceso, por lo que estos pueden decidir si acogen o no las recomendaciones que se les proporcionan por medio de las instancias distritales.

Por otro lado, tomando en cuenta el tamaño poblacional de las localidades, y aunque las normas no establecen un porcentaje mínimo de asistentes, se encontró que el nivel de participación de los ciudadanos en los EC es demasiado bajo en la actualidad para ser representativo de los intereses de las comunidades que las habitan ya que, en promedio no alcanza al 2,5\% de la población total de cada una (Fajardo et al. 2010). Por tanto, es necesario replantear la dinámica de estos EC, bien sea para redefinir la participación ciudadana mediante otros mecanismos, o para complementar estos escenarios con otros que permitan una mayor representatividad de los habitantes de las localidades.

En primera medida, además de la existencia de estos los EC sería conveniente considerar la posibilidad de llegar directamente a otro tipo de espacios como las escuelas, las universidades, las entidades públicas, las empresas, entre otros. Estas alternativas, podrían complementarse, además, con el uso de las TIC, como alternativa que permita recoger las iniciativas de individuos que no puedan hacer presencia en los escenarios físicos, pero que estén dispuestos a aportar en el proceso de construcción colectiva de los planes de desarrollo. Las TIC son herramientas que han venido cobrando cada vez mayor relevancia, no solo como mecanismo para recoger el aporte de soluciones a los problemas públicos por parte de los ciudadanos, sino además para la realización de trámites frente al Estado y la rendición de cuentas por parte de los responsables de administrar los asuntos públicos, entre otros (Magallón Rosa, 2014).

Asimismo, se deberá revisar el papel que deben cumplir otros actores en la planeación participativa, lo cual puede derivar en una fórmula distinta en términos tanto de la intervención de actores institucionales y privados considerados y no considerados actualmente, como de los escenarios de debate presenciales y no presenciales para identificar las propuestas ciudadanas (Estupiñán Villanueva et al., 2016; Cuadros Rodríguez et al., 2015; Valdés, 2011). La definición de los tiempos de la planeación, los actores con sus respectos roles, y los escenarios para el diálogo y el aporte de los ciudadanos, deberá derivar en un replanteamiento del diseño del proceso.

Como se mencionó antes, las metodologías de los EC definidas por los CPL son documentos que evidencian un nivel deficiente en la comprensión y la contribución de los distintos momentos del proceso. Por lo tanto, este rediseño deberá reconocer no solo la autonomía con la que ciertos actores deben ejercer su papel en la escena del diálogo social, sino, además, la de establecer un estándar mínimo en referencia al procedimiento en el que sean 
claros los momentos de la planeación local, así como los insumos, los actores participantes y las estrategias de diálogo más ajustadas a propósitos de cada uno de estos momentos.

Como resultado de la información recolectada en este estudio los momentos de la planeación en las localidades podrían incluir cuatro momentos distintos como son la identificación de problemas, la verificación, la priorización y la definición de alternativas de solución. En la actualidad existen diversas metodologías y herramientas tanto a nivel nacional como internacional (Ortegón, 2005) que podrían dar luces para que las autoridades centrales definan un instrumento estándar, adaptable a las necesidades de cada localidad. Este instrumento podría ofrecer diferentes alternativas a utilizar en relación con cada momento y su respectivo propósito dentro la planeación local. Lo anterior podría derivar, entre otros aspectos, en que los EC sean apenas un paso más dentro de una cadena o conjunto de estrategias de la planeación local. Pero, de acuerdo con este análisis, si éstos prevalecen, deberán ser reformulados en términos de sus propósitos, insumos, procedimientos y actores participantes, con el fin de garantizar su pertinencia.

\section{Referencias}

Colombia (1991, 13 de junio). Constitución Política de Colombia. Bogotá: Gaceta Constitucional No. 116.

Colombia (1992, 30 de abril). Acuerdo 6 de 1992. Por el cual se efectúa el reparto de competencia a que se refiere el artículo 322 de la Constitución Nacional, se adopta la organización administrativa de las Localidades en el D. C., se reglamenta su funcionamiento y se dictan otras disposiciones: Concejo Distrital de Bogotá.

Colombia (1993, 21 de julio). Decreto Ley 1421. Por el cual se dicta el régimen especial para el Distrito Capital de Santafé de Bogotá. Presidencia de la República de Colombia, Bogotá: Diario Oficial, No 40.958 del 22 de julio de 1993.

Colombia (1994, 15 de julio). Ley 152 de 1994. Por la cual se establece la Ley Orgánica del Plan de Desarrollo. Congreso de Colombia, Bogotá: Diario Oficial No. 41.450 del 19 de julio de 1994.

Colombia (1994, 9 de septiembre). Acuerdo 12 de 1994. Por el cual se establece el Estatuto de Planeación del Distrito Capital y se reglamenta la Formulación, la Aprobación, la Ejecución y la Evaluación del Plan de Desarrollo Económico y Social y de Obras Públicas del Distrito Capital de Santa Fe de Bogotá, y se dictan otras disposiciones complementarias. Concejo Distrital, Bogotá.

Colombia (2000, 6 de julio). Acuerdo 13 de 2000. Por el cual se reglamenta la participación ciudadana en la elaboración aprobación, ejecución, seguimiento, evaluación y control del Plan de Desarrollo Económico y Social para las diferentes Localidades que conforman el Distrito Capital y se dictan otras disposiciones. Concejo Distrital, Bogotá.

Colombia (2013, 5 de febrero). Ley 1617 de 2013. Por la cual se expide el Régimen para los Distritos Especiales. Congreso de Colombia, Bogotá: Diario Oficial No. 48.695 de 5 de febrero de 2013.

Colombia (2016, 10 de febrero). Circular Conjunta 005 del 10 de febrero de 2016. Lineamientos para la realización de Encuentros Ciudadanos y formulación de los Planes de Desarrollo Local 2017-2020. Alcaldía Mayor de Bogotá.

Bogotá Cómo Vamos (2017). Informe de Calidad de Vida en Bogotá. Bogotá: Fundación Corona, El Tiempo, Pontificia Universidad Javeriana, Cámara de Comercio de Bogotá.

Congreso de la República (1945). Acto Legislativo 01, Reformatorio de la Constitución. Diario Oficial. Año LXXX. N. 25769. 17, febrero, 1945. Pág. 1.

Cuadros Rodríguez, J. A., Arias García, S., \& Valencia Arias, A. (2015). La comunicación pública como estrategia orientadora en los procesos de participación ciudadana de los jóvenes. Encuentros, 13(1).

Cunill, N. (2004). Balance de la participación ciudadana en las políticas sociales. Propuesta de un marco analítico. Ziccardi A. (coord.) Participación ciudadana y políticas sociales en el ámbito local (p. 57-75). México: UNAMInstituto de Investigaciones sociales/Consejo Mexicano de Ciencias Sociales/INDESOL.

Diputació de Barcelona (2015). La participación ciudadana en la planificación estratégica territorial. Guías metodológicas para la planificación estratégica, 7. Oficina Técnica de Estrategias para el Desarrollo Económico y Oficina de Participación Ciudadana de la Diputación de Barcelona.

Duque-Cante, N. (2015). Características y alternativas de la organización político-administrativa de Bogotá. Revista de derecho: División de Ciencias Jurídicas de la Universidad del Norte, (44), 298-332. 
Estupiñán Villanueva, A., Molina Carmona, E., Flores Guevara, S., \& Martínez Bermeo, J. R. (2016). La participación digital en la construcción de la e-democracia y ciudadanía digital. RIDE. Revista Iberoamericana para la Investigación y el Desarrollo Educativo, 7(13), 126-146.

Fajardo, O., Moreno, N., \& Moreno, W. (2010). Encuentros ciudadanos 2008, formulación de planes de desarrollo locales. Instituto Distrital de la Participación Ciudadana IDPAC, Bogotá D.C.

Freire, P. (1979). Pedagogía del Oprimido. México: Siglo XXI.

Gómez, E., Vásquez, G., Lenti, A., Franco, L. M., Herrera, G., Aguirre, G. \& Giraldo, R. (2012). Planeación participativa. Realidades y retos. Universidad de Antioquia. Medellín, Colombia: Centro de Investigaciones Sociales y Humanas (CISH), Grupo de investigación cultura, política y Desarrollo social.

Harnecker, M., \& Bartolomé, J. (2015). Planificando desde abajo: una propuesta de planificación participativa descentralizada. Barcelona: El viejo topo.

Londoño, J. R. G. (2009). Pedagogía social o pedagogía de la participación ciudadana para una educación democrática. Unipluriversidad, 8(1).

Magallón Rosa, R. (2014). Tecnologías cívicas y participación ciudadana. Revista de estudios de juventud. Ediciones Injuve, 105, 53-79.

ONU (1948). Declaración Universal de Derechos Humanos. Ginebra, Suiza: Organización de las Naciones Unidas.

Ortegón, E. (2005). Metodología del marco lógico para la planificación, el seguimiento y la evaluación de proyectos y programas (Vol. 42). United Nations Publications.

PNUD. (2014). Informe auditoría a la democracia: más y mejor democracia para un Chile inclusivo. Santiago de Chile, Chile: LOM Ediciones, PNUD.

Prats Catalá, J. (2001). Gobernabilidad democrática para el Desarrollo Humano Marco Conceptual y Analítico. Instituciones y desarrollo, 10, 103-148.

Sandoval, C., Sanhueza, A., \& Williner, A. (2015). La planificación participativa para lograr un cambio estructural con igualdad: las estrategias de participación ciudadana en los procesos de planificación multiescalar. Manuales de la CEPAL. Santiago de Chile: Naciones Unidas.

SDP (2011). Guía para la formulación, ejecución, seguimiento y evaluación de los planes de desarrollo distrital y local. Bogotá: Secretaría Distrital de Planeación.

Velásquez, F (1986). Crisis municipal y participación ciudadana en Colombia. Revista Foro, 1, 16-25

Velásquez, F. \& González, E. (2004). La planeación participativa en Bogotá, análisis y propuestas. Bogotá, D. C.: Fundación Corona, Foro Nacional por Colombia.

Velásquez, F. \& González, E. (2003). ¿Qué ha pasado con la Participación Ciudadana en Colombia? Bogotá: Fundación Corona.

Williner, A. (2015). La construcción del Estado y los procesos de planificación multiescalar. En: Endara, G. (coord.) El rol del Estado: Contribuciones al debate, 189-221.

Editor: Paulo Nascimento Neto

Recibido: Oct. 23, 2018

Aprobado: Mayo 29, 2019 